\title{
A Hand-held OKP Chart for the Screening of Glaucoma: Preliminary Evaluation
}

\author{
B. E. DAMATO, J. CHYLA, E. McClURE, J. L. JAY, D. ALLAN \\ Glasgow
}

\begin{abstract}
Summary
Oculo-kinetic perimetry (OKP) uses controlled movements of the patient's eye to position a static test stimulus in the visual field. The test chart consists of a white tangent screen with 23 numbered fixation points located eccentrically at strategic points in relation to a central black spot, which is the test stimulus. As the patient looks at each number in turn, the spot stimulates the retinal areas that are most likely to show early glaucomatous damage. The result is positive if one or more numbers make the spot disappear. Ninety-eight eyes of 54 patients attending a hospital glaucoma clinic were tested with a prototype OKP glaucoma screener and by conventional perimetry. Visual fields plotted conventionally were independently categorised as normal, equivocal and glaucomatous by the perimetrist and the ophthalmologist and these results were further categorised as normal, equivocal or glaucomatous according to whether they were considered abnormal by none, one or both of the observers respectively. The OKP test was positive in

(i) $93 \%$ of 27 eyes with glaucomatous defects,

(ii) $69 \%$ of 32 eyes with equivocal loss,

(iii) $41 \%$ of 39 eyes without previously recognised field loss but having ocular hypertension, a suspicious disc, or contralateral glaucoma,

(iv) $9 \%$ of 116 eyes from 60 age matched persons accompanying the patients to hospital.

If the results were considered in terms of patients not eyes, the OKP test was positive in $85 \%$ individuals with glaucomatous field loss in their worse eye and in $12 \%$ of the controls. A hand-held OKP chart testing a small set of points and using a constantly exposed stimulus could be a useful tool for the detection of glaucoma in the community.
\end{abstract}

There is scope for improvement in the screening of glaucoma. This disease often causes extensive loss of vision before it is detected, ${ }^{1}$ and paradoxically, most individuals referred to the ophthalmologist with suspected glaucoma are incorrectly diagnosed. ${ }^{2}$ In ideal conditions, perimetry can detect glaucomatous eyes with high levels of sensitivity and specificity; ${ }^{3,4}$ in practice, however, the role of conventional perimetry is limited by the cost of the equipment, and the time and expertise required. ${ }^{5,6}$

Oculo-kinetic perimetry (OKP) is uniquely suited to screening for glaucoma because it is both simple and inexpensive, to the extent that patients can examine themselves without assistance using a disposable paper chart. ${ }^{7}$ This is achieved by using controlled movements of the patient's eye to place an inert test stimulus at preselected points in the visual 
field, thereby eliminating the need for movement of the test stimulus and for ensuring that the eye is stationary. A previous study has shown that glaucomatous visual fields plotted with a general purpose, 100-point chart show good correlation with sophisticated conventional perimetry. ${ }^{8}$ Although suitable for general use, this chart is not appropriate for screening purposes because it is too time consuming, taking about four minutes to examine a normal eye, and because it requires skilled interpretation of the results. In another investigation, we identified a small subset of points on this chart that are missed specifically by glaucomatous eyes, and predicted that an OKP chart testing only six points would detect $85 \%$ of glaucomatous eyes with a false positive rate of $10 \%$. $^{\prime}$

The aim of the present study is to determine whether glaucomatous visual field defects can be detected using a small chart with a working distance of $40 \mathrm{~cm}$ and a constantly exposed stimulus, unlike the previous study in which a one metre chart was used and in which the stimulus was intermittently exposed.

\section{Patients and methods}

The glaucomatous eyes were selected from patients attending a hospital glaucoma clinic and the control eyes from friends and relatives accompanying these patients. Eyes were excluded from the study

(i) if the visual acuity was less than $6 / 18$,

(ii) if the visual field was severely constricted

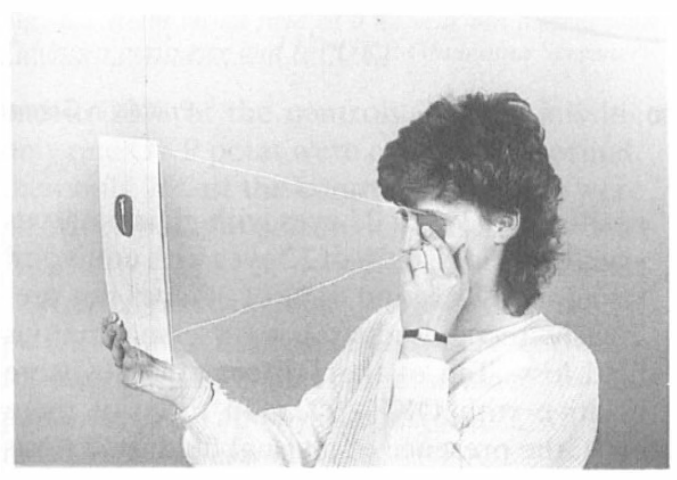

Fig. 1. The prototype OKP Glaucoma Screener, consisting of a double-sided card with a chart on either side, for the left and right eyes respectively. An eyepatch was attached to the chart by a ribbon of the appropriate length to maintain the eye at the correct distance from the chart. (ie, absolute field loss to less than 5 degrees of fixation in all quadrants),

(iii) in the case of control eyes, if there was any history suggestive of ocular disease. Individuals who were obviously very frail were not invited to participate in the study.

The prototype OKP glaucoma screener consisted of a double-sided card with a chart on either side, for the left and right eyes respectively (Fig. 1). Each chart consisted of a white tangent screen, with a central black spot (the test stimulus) and 23 numbered fixation points peripherally, which were located so as to test the central field up to $12.5^{\circ}$ superiorly and $15^{\circ}$ nasally and inferiorly (Fig. 2). An eyepatch was attached to the chart by a ribbon of the appropriate length to maintain the eye at the correct distance from the chart. The patients were asked to look at each number in turn and to identify the numbers that made the spot disappear. The result was considered positive if one or more points were missed. The subjects received their instructions orally or in writing and were left to perform the examination without assistance. They were kept under observation, however, and corrected if seen to be doing the test incorrectly. The time taken to examine each eye was measured and, in a sample of individuals, the time taken to explain the test procedure was also measured.

Conventional perimetry was performed by optometrists using the Friedmann Mark II Visual Field Analyser, the Tubingen perimeter or the Dicon AP 3000 automated perimeter. The results were categorised independently by the optometrist responsible for the perimetry (EM) and by the ophthalmologist in charge of the glaucoma clinic $(\mathrm{JJ})$ as normal, equivocal or glaucomatous. The two independent assessments were subsequently merged into the following categories: normal (ie, field categorised as normal or equivocal by both observers); equivocal (ie, field categorised as glaucomatous by one observer only); and glaucomatous (ie, field categorised as glaucomatous by both observers).

\section{Results}

There were 98 eyes from 54 patients attending the glaucoma clinic (48 male: 50 female; 


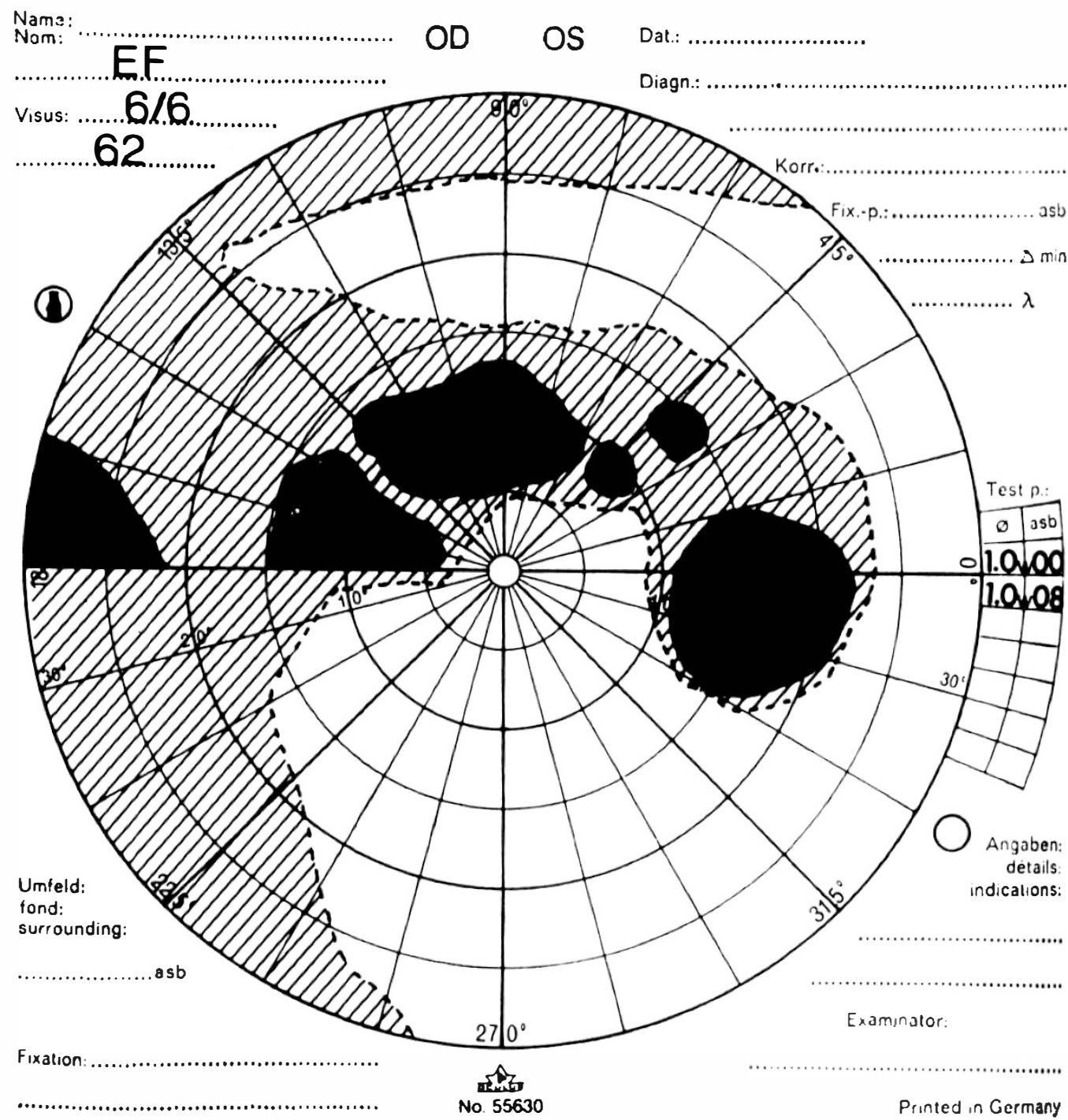

Fig. 2it.

Mean age 64.2 yrs; $S D=10.5)$ and 116 eyes from 60 accompanying persons (52 male: 65 female; Mean age 63.2 yrs; $\mathrm{SD}=9.9$ ). The two groups did not differ significantly in age $(\mathrm{t}$ test; $0.2<\mathrm{p}<0.5$ ).

The independent and merged categorisations of the conventional visual fields are shown in Figure 3. The ophthalmologist tended to grade the visual fields less severely than the optometrist.

The results of the OKP glaucoma screener are shown in Figure 4. In the patients attending the glaucoma clinic, the OKP test was positive in $93 \%$ of 27 eyes with glaucomatous visual field loss, $69 \%$ of 32 eyes with equivocal visual field loss, and $41 \%$ of 39 eyes not previously recognised as having glaucomatous field loss. Ten of the 116 control eyes were positive with OKP $(9 \%)$. In three of these eyes, the presence of a visual field defect was subsequently confirmed by conventional perimetry and found to be due to cataract or macular degeneration. If the results were considered in terms of individuals instead of eyes, OKP was positive in $85 \%$ of individuals with glaucomatous defects in their worse eye 


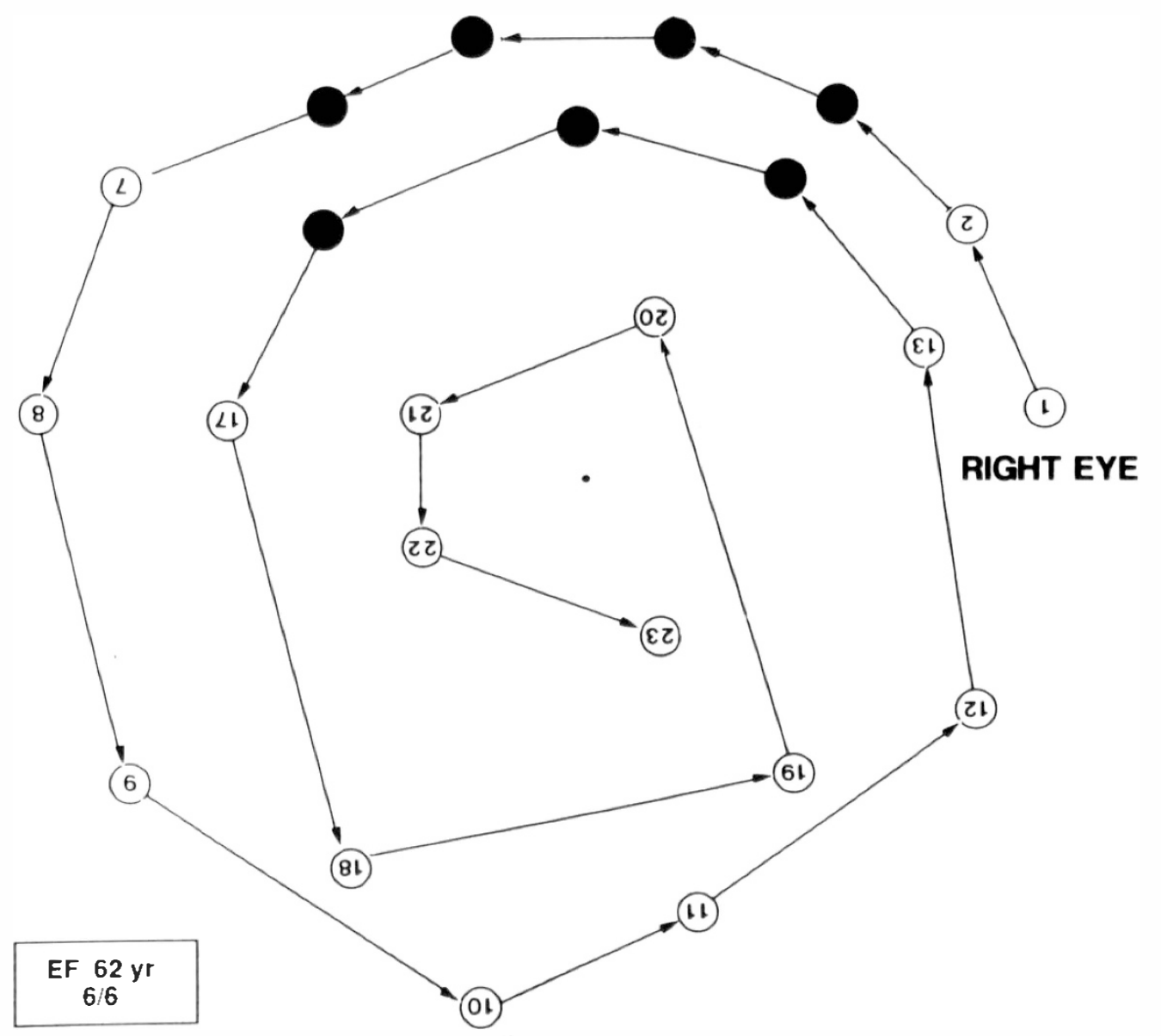

Fig. 2 b.

Fig. 2. Right visual field of a 62 year old patient with a superior arcuate visual field defect plotted with the (i) Tubingen perimeter and (ii) OKP Glaucoma Screener.

and in $12 \%$ of the controls. If eyes missing only one OKP point were considered normal, then only $7 \%$ of the control individuals were positive, but the sensitivity was reduced to $73 \%$.

Written instructions were given to 38 patients and 43 controls and were correctly followed without further assistance by 24 patients $(63 \%)$ and by 31 controls $(72 \%)$. The most frequent errors were

(i) performing the test too quickly,

(ii) looking directly at the test stimulus,

(iii) holding the chart too close to the eye.

The patients took. longer to perform the OKP examination than the controls, requiring a mean of 78 secs $(\mathrm{SD}=54$ secs $)$ as against
56 secs for the controls $(\mathrm{SD}=39$ secs) ( $\mathrm{t}$ test; $\mathrm{p}<0.001)$.

\section{Discussion}

The sensitivity and specificity achieved in this study are similar to those predicted by our previous investigation, in which a one-meter OKP chart was used, with constant supervision and with the stimulus covered and uncovered. ${ }^{9}$ The findings of the present study are also in keeping with results obtained by other workers using conventional perimeters. ${ }^{3,12}$ Such a comparison can only be approximate, since results depend on the severity of the visual field loss in the sample and on the exclusion criteria followed. If we had 


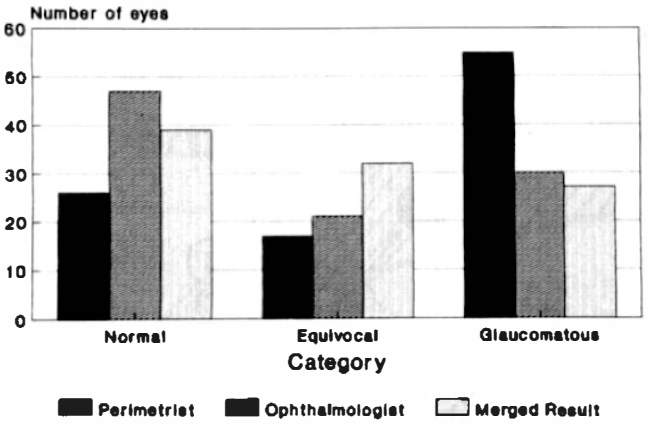

Fig. 3. Results of conventional perimetry as categorised independently by the optometrist and ophthalmologist as compared to merged results.

pre-screened our controls, like some other authors who excluded $40 \%$ of their sample, ${ }^{4}$ we would have achieved a false positive rate of only $5 \%$ eyes, or $8 \%$ individuals. There is scope for further studies, conducted by independent workers and under more natural working conditions. In addition, it would be more informative to present the results in terms of patients, not just eyes, in studies of this kind.

The $41 \%$ positive rate in the group of 'normal' eyes, from patients attending the glaucoma clinic requires explanation. It is possible that the black stimulus on the OKP chart has detected subtle defects that were missed by the conventional light stimuli. Alternatively, patients who were expecting to have a visual field defect may have reacted differently from the volunteers presuming themselves to be healthy. Since the patients and volunteers were selected and supervised during the examination, observer bias may have contributed to the results.

This study drew attention to a number of shortcomings in the design of the chart, which limited self-examination. These have since been rectified by

(i) using a rigid side-arm, instead of the ribbon, to ensure that the chart is held at the correct distance from the eye;

(ii) printing an improved set of instructions on the chart itself so that they can be read conveniently during the examination;

(iii) advising that the test be repeated and considering only consistently missed points to be significant. Other studies have shown that perimetric results are more reliable when the examination is repeated. ${ }^{10,11}$

The OKP glaucoma screener may have new applications in the screening of glaucoma. Firstly, this test could be used to screen individuals who might not otherwise be examined using conventional methods. Inexpensive disposable charts could, for example, be distributed to specific target groups, such as relatives of individuals with glaucoma, or the staff of a large hospital for factory, for selfexamination. The feasibility of 'remote screening' is already being investigated, with encouraging preliminary results. Secondly, the OKP Glaucoma Screener could be used by primary health care workers in the community as an adjunct to tonometry and ophthalmoscopy; this may help the health worker to decide whether or not to refer an individual with suspicious discs or borderline intraocular pressures for specialist opinion and enhance the detection of low-tension glaucoma. Finally, reliable glaucoma suspects under the care of the ophtalmologist could use these charts for self-examination at home thereby allowing the frequency of clinic visits to be reduced.

It is hoped that the OKP glaucoma screener will complement conventional perimeters rather than compete with them. By making visual field examination more widely available, this tool could provide a new impetus to the screening of glaucoma.

We gratefully acknowledge the financial assistance of the Ross Foundation for the Prevention of Blind-

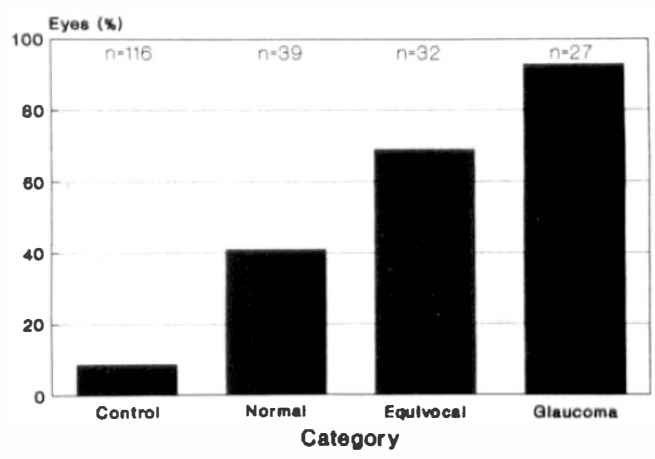

Fig. 4. Results of OKP Glaucoma Screener in control eyes of accompanying persons, and patients attending a glaucoma clinic with normal, equivocal, and glaucomatous visual fields respectively. 
ness (Scotland) and the artwork of Mrs D Aitken and Mrs S Cameron.

\section{References}

' Jay JL and Murray SB: Early trabeculectomy versus conventional management in primary open angle glaucoma. Br J Ophthalmol 1988, 72: 881-9.

${ }^{2}$ Harrison RJ, Wild JM, Hobley AJ: Referral patterns to an ophthalmic outpatient clinic by general practitioners and ophthalmic opticians and the role of these professionals in screening for ocular disease. Br Med J 1988, 297: 1162-7.

${ }^{3}$ Henson DB, Chauhan BC, Hobley A: Screening for glaucomatous visual field defects: the relationship between sensitivity, specificity and the number of test locations. Ophthal Physiol Opt 1988, 8: 123-7.

${ }^{4}$ Enger $\mathrm{C}$ and Sommer A: Recognizing glaucomatous field loss with the Humphrey STATPAC. Arch Ophthalmol 1987, 105: 1355-7.

${ }^{5}$ Keltner JL and Johnson CA: Current status of automated perimetry. Is the ideal automated perimeter available? Arch Ophthalmol 1986, 104: 347-9.
${ }^{6}$ Gottleib LK, Schwartz B, Pauker SG: Glaucoma Screening. A cost-effectiveness analysis. Surv Ophthalmol 1983, 28: 206-26.

${ }^{7}$ Damato BE: Oculo-Kinetic Perimetry: a simple visual field test for use in the community. $\mathrm{Br} J$ Ophthalmol 1985, 69: 927-31.

${ }^{8}$ Alvarez E, Damato BE, Jay JL, McClure E: Comparative evaluation of oculokinetic perimetry and conventional perimetry in glaucoma. $\mathrm{Br} J \mathrm{Oph}$ thalmol 1988, 72: 258-62.

${ }^{9}$ Damato BE, Ahmed J, Allan D, McClure E, Jay JL: The detection of glaucomatous visual field defects by oculo-kinetic perimetry: Which points are best for screening? Eye 1989, 3: 727-31.

${ }^{10}$ Rock WJ, Drance SM, Morgan RW: Visual field screening in glaucoma. An evaluation of the Armaly technique for screening glaucomatous visual fields. Arch Ophthalmol 1973, 89: 287-90.

${ }^{11}$ Heijl A, Lindgren G, Olsson J: The effect of perimetric experience in normal subjects. Arch Ophthalmol 1989, 107: 81-6.

${ }^{12}$ Rabin S, Kolesar P, Podos SM, Wilensky JT: A visual field screening protocol for glaucoma. Am J Ophthalmol 1981, 92: 530-5. 